\title{
Estado nutricional foliar de tres cultivares de mango fertilizados con abonos orgánicos
}

\author{
Nutritional foliar status of three mango cultivars \\ fertilized with organic amendments
}

\author{
Nain Peralta-Antonio ${ }^{1 *}$, A. Enrique Becerril-Román², \\ Andrés Rebolledo-Martínez ${ }^{1}$, David Jaén-Contreras ${ }^{2}$
}

\section{RESUMEN}

El trabajo se realizó con el objetivo de conocer la influencia de los fertilizantes orgánicos lombricompost, bocashi, gallinaza a 10 y $5 \mathrm{t} \mathrm{ha}^{-1}$ y las dosis 230-0-300 y 230-0-0 g de NPK por árbol sobre la nutrición foliar de mango 'Manila Cotxtla 2' ('MC2'), 'Tommy Atkins' ('TA') y 'Ataulfo' ('A'). Previo a floración y previo a cosecha se midieron las concentraciones foliares de N, K, $\mathrm{Ca}, \mathrm{Mg}, \mathrm{Zn}, \mathrm{Fe}, \mathrm{Mn}$ y Cu. Los resultados indican que, previo a floración la mayor concentración de N fue en 'TA', de Ca en 'A', y de Zn, Fe y Mn en 'MC2'; previo a cosecha, 'TA' indujo más concentración de $\mathrm{K}$ y Zn, 'A' superior en Ca, $\mathrm{Mg}$ y Cu, 'MC2' mayor concentración de N. Entre fuentes de fertilización, lombricompost $5 \mathrm{t} \mathrm{ha}^{-1}$, superó a 230-0-300 g de N-P-K por árbol en concentración de $\mathrm{Ca}$ (previo a floración) y $\mathrm{Cu}$ (previo a cosecha), mientras que esta dosis superó a todos los fertilizantes orgánicos y testigo en la concentración de Mn (previo a cosecha). Los valores de $\mathrm{N}$ (1,22-1,38 g $\left.100 \mathrm{~g}^{-1} \mathrm{~ms}\right)$ se encuentran en el rango adecuado, $\mathrm{K}$

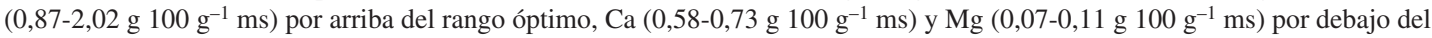
rango óptimo, mientras que $\mathrm{Zn}\left(22,9-35,8 \mathrm{mg} \mathrm{kg}^{-1} \mathrm{~ms}\right), \mathrm{Fe}\left(70,3-102,5 \mathrm{mg} \mathrm{kg}^{-1} \mathrm{~ms}\right), \mathrm{Mn}\left(49,9-89,9 \mathrm{mg} \mathrm{kg}^{1} \mathrm{~ms}\right)$ y Cu (11,4-19,7 $\mathrm{mg} \mathrm{kg}^{-1} \mathrm{~ms}$ ) se encuentran en rangos adecuados. Se concluye que la composición nutrimental del follaje presentó variaciones dentro de cada cultivar y entre cultivares, la cantidad de nutrimentos removidos también está influenciado por el cultivar y las condiciones del cultivo; a excepción de $\mathrm{Cu}$ y Mn, las fuentes orgánicas y minerales indujeron comportamientos nutrimentales foliares similares.

Palabras clave: Mangifera indica, fertilizantes orgánicos, nutrientes, N, K.

\section{ABSTRACT}

The work was performed in order to determine the influence of organic fertilizer Vermicompost, Bokashi, Gallinaza to 10 and 5 $t$ ha $\mathrm{a}^{-1}$ and doses 230-0-300 (M1) and 230 0-0 NPK g tree ${ }^{-1}$ on the foliar nutritional status of Manila Cotxtla 2 ('MC2'), Tommy Atkins ('TA') and Ataulfo ('A') mango cultivars. Prior to flowering and before harvest, foliar concentrations of $\mathrm{N}, \mathrm{K}, \mathrm{Ca}, \mathrm{Mg}, \mathrm{Zn}$, $\mathrm{Fe}, \mathrm{Mn}$ and $\mathrm{Cu}$ were measured. The results indicate that, prior to flowering highest concentration of $N$ was in 'TA', Ca in 'A', and $\mathrm{Zn}, \mathrm{Fe}$ and Mn in 'MC2'. Prior to harvest, 'TA' induced more concentration of $\mathrm{K}$ and $\mathrm{Zn}$, 'A' higher in $\mathrm{Ca}$, $\mathrm{Mg}$ and $\mathrm{Cu}$, highest concentration of $N$ in 'MC2'. Among sources of fertilization, vermicompost $5 \mathrm{th} \mathrm{a}^{-1}$, outperformed to 230-0-300 $\mathrm{g}$ of NPK per tree in Ca concentration (before flowering) and $\mathrm{Cu}$ (pre-harvest), whereas this dose exceeded all organic fertilizers and control

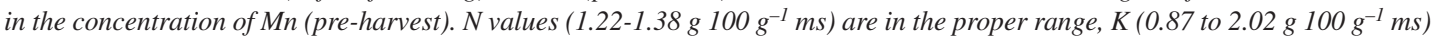

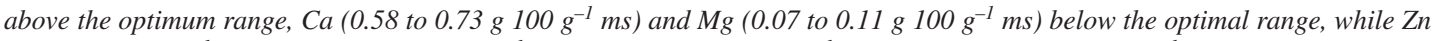
(22.9-35.8 $\left.\mathrm{mg} \mathrm{kg}^{-1} \mathrm{~ms}\right), \mathrm{Fe}\left(70.3-102.5 \mathrm{mg} \mathrm{kg} \mathrm{g}^{-1} \mathrm{~ms}\right), \mathrm{Mn}\left(49.9-89.9 \mathrm{mg} \mathrm{kg}^{-1} \mathrm{~ms}\right)$ and $\mathrm{Cu}\left(11.4-19.7 \mathrm{mg} \mathrm{kg}^{-1} \mathrm{~ms}\right)$ are in appropriate ranges. It is concluded that the nutritional composition of foliage presented variations within each cultivar and between cultivars, the amount of nutrients removed is also influenced by cultivar and growing conditions; except for Cu and Mn, organic and mineral sources induce similar behavior in the foliar nutritional status.

Key words: Mangifera indica, organic fertilizers, nutrients, $N, K$.

\section{Introducción}

En sus diferentes modalidades la incorporación de materia orgánica al suelo, en las unidades de producción frutícolas, son alternativas para la nutrición de los árboles, entre las opciones se pueden considerar: compostas, fertilizantes fermentados y residuos de cosechas. Estos residuos orgánicos son

1 Instituto Nacional de Investigaciones Forestales Agrícolas y Pecuarias (INIFAP), km 34,5 Carretera Federal Veracruz-Córdoba, Medellín de Bravo, Vera Cruz, México, México.

2 Colegio de Postgraduados, km 36.5 Carretera México-Texcoco, Texcoco, Edo. de México, México.

* Autor para correspondencia: peralta.nain@inifap.gob.mx

Fecha de Recepción: 16 Marzo, 2015.

Fecha de Aceptación: 29 Mayo, 2015. 
liberadores de nutrientes de forma lenta, con lo que se disminuye el riesgo de pérdida por lixiviación y a su vez estimula la presencia y diversidad de organismos en la rizosfera de los árboles. Para el cultivo de mango (Mangifera indica L.) existen pocos antecedentes sobre la nutrición orgánica del cultivo de mango, en particular si se desea conocer su efecto sobre el estado nutrimental de las hojas. Entre los estudios realizados se encuentra el de Moyin-Jesu y Adeofun (2008), en Nigeria, que en condiciones de vivero utilizaron como fuente de fertilización la ceniza de palma de aceite, restos de cereal, estiércol de pavo, estiércol de aves de corral (en dosis de $8 \mathrm{t} \mathrm{ha}^{-1}$ ), la dosis mineral 1515-15 de NPK (400 kg ha ${ }^{-1}$ ), comparándolos con un testigo; observaron que la aplicación conjunta de ceniza de palma de aceite y estiércol de aves de corral indujo mayor concentración de $\mathrm{K}, \mathrm{Ca}$ y $\mathrm{Mg}$ que la fertilización mineral y el testigo. En Brasil Silva et al. (2013) evaluaron en el cv. Tommy Atkins tres tipos de compostas en dosis de $0,5 \mathrm{y}$ $10 \mathrm{t} \mathrm{ha}^{-1}$, los resultados no mostraron diferencias estadísticas en los contenidos de N, P, K y Ca de las hojas, aunque sí existió en el rendimiento de frutos. En otros frutales también existen reportes de la fertilización orgánica; en México Tapia et al. (2014) evaluaron en aguacate Hass la aplicación de fermentos orgánicos, derivados de pescado, composta, microorganismos, lombricompost y lo compararon con una dosis mineral convencional; en los resultados notaron que los fermentos orgánicos mostraron mayor concentración foliar de $\mathrm{N}$ y K en comparación con la dosis mineral. Lo descrito anteriormente demuestra la capacidad de las fuentes orgánicas para mejorar la fertilidad del suelo y reflejarlo en la condición nutrimental de las hojas, motivo por el que se desarrolló la presente investigación, que tuvo como objetivo determinar el efecto de la fertilización orgánica edáfica sobre la concentración nutrimental de las hojas, de tres cultivares de mango.

\section{Materiales y Métodos}

El trabajo se desarrolló en el Campo Experimental Cotaxtla (INIFAP), localizado a $18^{\circ} 56^{\prime} 13^{\prime \prime} \mathrm{N}$ y $96^{\circ} 11$ ' $38^{\prime \prime} \mathrm{W}$, en Veracruz, México. El suelo es vertisol pélico con pendiente de terreno menor al $3 \%$, las características del suelo durante el periodo de evaluación (2011) se muestran en la Tabla 1.

Los cultivares de mango utilizados fueron: 'Manila Cotaxtla 2' ('MC2'), 'Tommy Atkins' ('TA') y 'Ataulfo' ('A'), establecidos en campo en noviembre de 2006, con distanciamientos de $6 \mathrm{~m} \times 2,5 \mathrm{~m}$. Las fuentes de fertilización fueron: lombricompost, bocashi y gallinaza en dosis de 5 y $10 \mathrm{t} \mathrm{ha}^{-1}$ (equivalente a 7,5 y $15 \mathrm{~kg} \mathrm{arbol}^{-1}$ ), dos dosis minerales: 230-0-300 g de NPK árbol ${ }^{-1}$ y 230-0-0 g de NPK árbol ${ }^{-1}$ y un testigo. Las características de los diferentes fertilizantes orgánicos se presentan en la Tabla 2. La fertilización de los árboles se inició en septiembre de 2009; durante ese año las aplicaciones se realizaron en la periferia de la copa, para lo cual se efectuaron excavaciones de aproximadamente $20 \mathrm{~cm}$ de ancho por $10 \mathrm{~cm}$ de profundidad, en donde se depositaron los fertilizantes y se procedió

Tabla 1. Características de $\mathrm{pH}$, materia orgánica (MO) y nutrientes $\left(\mathrm{mg} \mathrm{kg}^{-1}\right)$ en suelo plantado con tres cultivares de mango, manejados con diferentes fuentes de fertilización, en las profundidades de 0-40 cm, en Veracruz, México, durante 2011.

\begin{tabular}{cccccccccccc}
\hline \multirow{2}{*}{$\mathrm{Ph}$} & $\mathrm{MO}(\%)$ & \multicolumn{10}{c}{$\mathrm{mg} \mathrm{kg}^{-1}$} \\
\cline { 3 - 13 } & & $\mathrm{N}$ & $\mathrm{P}$ & $\mathrm{K}$ & $\mathrm{Ca}$ & $\mathrm{Mg}$ & $\mathrm{Cu}$ & $\mathrm{Zn}$ & $\mathrm{Fe}$ & $\mathrm{Mn}$ \\
\hline 6,5 & 2,0 & 17,1 & 18,4 & 303,1 & 2568,2 & 461,8 & 1,6 & 1,5 & 23,1 & 15,3 \\
\hline
\end{tabular}

Tabla 2. Características de tres fertilizantes orgánicos empleados en la nutrición de tres cultivares de mango, en Veracruz, México.

\begin{tabular}{lccccccccccc}
\hline \multirow{2}{*}{ Fuentes orgánicas } & $\mathrm{pH}$ & $\mathrm{MO}(\%)$ & \multicolumn{1}{c}{$\mathrm{mg} \mathrm{kg}^{-1}$} \\
\cline { 3 - 12 } & & & $\mathrm{N}$ & $\mathrm{P}$ & $\mathrm{K}$ & $\mathrm{Ca}$ & $\mathrm{Mg}$ & $\mathrm{Fe}$ & $\mathrm{Cu}$ & $\mathrm{Zn}$ & $\mathrm{Mn}$ \\
\hline Gallinaza & 7,17 & 34,14 & 3220 & 10613 & 13673 & 1362 & 1361 & 64,72 & 31,78 & 27,2 & 66,28 \\
Lombricompost & 4,74 & 6,68 & 3115 & 214,21 & 3466 & 9010 & 1313 & 94,31 & 1,9 & 9,99 & 37,27 \\
Bokashi & 7,31 & 28,81 & 322 & 894 & 12356 & 6020 & 2713 & 35,77 & 4,06 & 19,11 & 53,72 \\
\hline
\end{tabular}


a cubrirlos. A partir de 2010, los fertilizantes se distribuyeron uniformemente en toda el área bajo la copa, en los primeros $10 \mathrm{~cm}$ de profundidad y se cubrió con suelo. Durante la época de estiaje (diciembre a mayo) se aplicaron a todos los árboles láminas de riego de $54 \mathrm{~mm}$ rodados cada 20 días. El control de maleza se realizó de forma mecánica y manual, bimestralmente.

Durante el periodo que comprendió el estudio, el comportamiento del clima se caracterizó por mostrar temperaturas mínimas que oscilaron entre $14,0{ }^{\circ} \mathrm{C}$ (diciembre 2010) y $29,0^{\circ} \mathrm{C}$ (enero 2011), temperaturas máximas de $26,6^{\circ} \mathrm{C}$ (diciembre 2010) a $37,7^{\circ} \mathrm{C}$ (enero 2011 ) y una temperatura media de $19,8^{\circ} \mathrm{C}$ (diciembre 2010) a $33,0^{\circ} \mathrm{C}$ (enero 2011); la humedad relativa fluctuó entre $74,2 \%$ y $87,9 \%$, con excepción de abril, mayo, junio 2011, donde osciló entre $27,2 \%$ y $30,2 \%$; respecto de la precipitación pluvial, en 2010 marzo fue el mes más seco, ya que no se presentaron precipitaciones, mientras que en septiembre del mismo año se precipitó la mayor cantidad de lluvia $(584,6 \mathrm{~mm})$, en 2011, febrero, marzo y abril fueron los meses de sequía, mientras que de junio a noviembre de ese año se precipitó la mayor cantidad de lluvia (323 mm), como se muestra en la Figura 1.

Se realizaron determinaciones nutrimentales foliares, para ello se colectaron al azar 20 hojas por árbol. Las hojas se colectaron en enero de 2010, previo a floración y en mayo previo a cosecha, posteriormente en 2011 las colectas fueron en diciembre previo a floración y abril previo a cosecha. Las hojas colectadas fueron trasladadas al laboratorio, se lavaron con agua corriente y fueron enjuagadas con agua desionizada, posteriormente fueron secadas en una estufa con ventilación forzada a $70{ }^{\circ} \mathrm{C}$ hasta alcanzar peso constante. La molienda fue mecánica con un molino tipo wiley con malla de 30 micras. Posteriormente las muestras fueron tratadas mediante digestión vía húmeda con una mezcla de ácidos nítrico y perclórico en relación 2:1. Para medir la concentración de N se utilizó el método Kjeldahl, el K se midió por flamometría, para la cuantificación de los elementos $\mathrm{Ca}, \mathrm{Mg}$, $\mathrm{Mn}, \mathrm{S}, \mathrm{Cu}$, y Fe se utilizó un espectrofotómetro de absorción atómica.

Para todas las variables, el diseño utilizado fue completamente al azar con arreglo en parcelas divididas, siendo la parcela grande el cultivar y la parcela chica las fuentes de fertilización. Se utilizaron tres repeticiones, considerando un árbol como unidad experimental. Análisis de varianza y separación de medias de Tukey (HSD) con un nivel de $\mathrm{P} \leq 0,05$ fueron realizados mediante el programa estadístico SAS versión 9,2 (SAS Institute Inc., 2007).

\section{Resultados y Discusión}

\section{Cultivares de mango}

Previo a floración, 'TA' mostró mayor concentración de $\mathrm{N}$ que 'A'; mientras que, previo a cosecha, 'TA' y 'A' fueron superados por 'MC2'. Respecto de K, antes de la floración, los tres cultivares de mango mostraron igualdad estadística; en tanto que, antes de la cosecha, 'TA' y 'A' sobrepasaron a 'MC2'. En cuanto a $\mathrm{Ca}$, en las dos fechas de muestreo, ' $\mathrm{A}$ ' superó a 'MC2' y 'TA'. Con relación a Mg, antes de la floración, los tres cultivares fueron iguales, y previo a cosecha, 'A' superó a 'TA' (Tabla 3). Respecto de $\mathrm{Zn}$, antes de la floración, 'M' superó a 'TA' y 'A', y previo a cosecha, nuevamente 'MC2' superó a 'TA'.

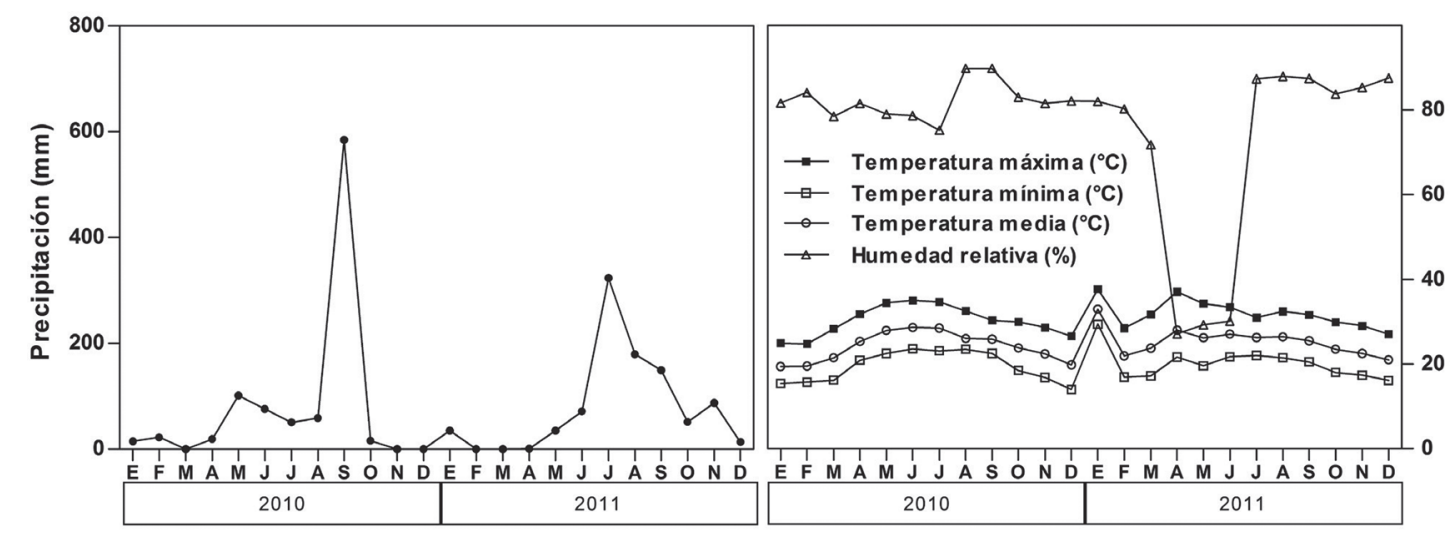

Figura 1. Comportamiento del clima en la región costera central de Veracruz, México, durante dos años consecutivos. 


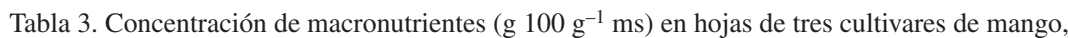
previo a floración, en Veracruz, México, durante el ciclo de producción 2011.

\begin{tabular}{lcccccccc}
\hline \multirow{2}{*}{ Cultivar } & \multicolumn{3}{c}{ Previo a floración } & \multicolumn{4}{c}{ Previo a cosecha } \\
\cline { 2 - 9 } & $\mathrm{N}$ & $\mathrm{K}$ & $\mathrm{Ca}$ & $\mathrm{Mg}$ & $\mathrm{N}$ & $\mathrm{K}$ & $\mathrm{Ca}$ & $\mathrm{Mg}$ \\
\hline Manila Cotaxtla 2 & $1,29 \mathrm{ab}$ & $1,20 \mathrm{a}$ & $0,60 \mathrm{~b}$ & $0,09 \mathrm{a}$ & $1,48 \mathrm{a}$ & $1,29 \mathrm{~b}$ & $0,57 \mathrm{~b}$ & $0,09 \mathrm{ab}$ \\
Tommy Atkins & $1,36 \mathrm{a}$ & $1,03 \mathrm{a}$ & $0,59 \mathrm{~b}$ & $0,08 \mathrm{a}$ & $1,18 \mathrm{~b}$ & $2,19 \mathrm{a}$ & $0,66 \mathrm{~b}$ & $0,08 \mathrm{~b}$ \\
Ataulfo & $1,22 \mathrm{~b}$ & $1,09 \mathrm{a}$ & $0,71 \mathrm{a}$ & $0,08 \mathrm{a}$ & $1,22 \mathrm{~b}$ & $2,02 \mathrm{a}$ & $0,83 \mathrm{a}$ & $0,10 \mathrm{a}$ \\
CV & 10,8 & 27,5 & 17,1 & 16,9 & 5,9 & 17,8 & 17,7 & 15,1 \\
\hline
\end{tabular}

Columnas con la misma letra son estadísticamente iguales (Tukey $\alpha=0,05$ ).

Para el caso de Fe, en los dos muestreos, 'MC2' superó 'TA' y 'A'. En cuanto a Mn, previo a floración, 'MC2' continuó superando a los otros dos cultivares y se comportó estadísticamente igual a ellos previo a la cosecha. Previo a floración, las concentraciones de $\mathrm{Cu}$ de 'MC2' y 'A' fueron mayores que los de 'TA', y antes de la cosecha fue 'A' quien superó a 'TA' y 'MC2' (Tabla 5). En forma decreciente, previo a floración la concentración de nutrientes en los tres cultivares fue: $\mathrm{N}>\mathrm{K}>\mathrm{Ca}>\mathrm{Mn}>\mathrm{Mg}>\mathrm{Fe}>\mathrm{Zn}>\mathrm{Cu}$ $\mathrm{y}$ previo a cosecha se presentaron pequeñas variaciones: $\mathrm{N}>\mathrm{K}>\mathrm{Ca}>\mathrm{Fe}>\mathrm{Mg}>\mathrm{Mn}>\mathrm{Zn}>\mathrm{Cu}$ ('MC2'), $\mathrm{K}>\mathrm{N}>\mathrm{Ca}>\mathrm{Fe}>\mathrm{Mg}>\mathrm{Mn}>\mathrm{Zn}>\mathrm{Cu}$ ('TA') y $\mathrm{K}>\mathrm{N}>\mathrm{Ca}>\mathrm{Mg}>\mathrm{Mn}>\mathrm{Fe}>\mathrm{Zn}>\mathrm{Cu}$ ('A'). Los resultados no muestran el mismo patrón nutrimental antes de floración y antes de la cosecha, en los tres cultivares de mango, lo que se atribuye a diferentes factores, como: la alternancia del árbol, etapa fenológica del árbol, presencia de flores o frutos en los brotes muestreados, edad de la hoja y su ubicación dentro del árbol. Ya que Tahir et al. (2003) indican que la concentración de nutrimentos en hojas de brotes productivos es menor después de la cosecha y se incrementa después de la diferenciación, en tanto que, en brotes no productivos, la evolución en la concentración de nutrimentos de forma inversa; Chadha et al. (1980) reportan que los nutrimentos tienen diferentes patrones en el transcurso del tiempo, algunos pueden incrementar su concentración $(\mathrm{Ca}$, $\mathrm{Mg}, \mathrm{S}, \mathrm{Fe}, \mathrm{Mn}, \mathrm{Zn}$ ), disminuir (P, K) o mantenerse sin mucha variación (N); Castro-López et al. (2012) al monitorear el comportamiento nutrimental foliar de los cultivares Kent, Ataulfo y Tommy Atkins apreciaron que flujos de primavera mostraron los valores más bajos en cosecha y fueron incrementándose a la llegada de la antesis y la formación de frutos, mientras que hojas generadas en verano expusieron sus menores contenidos durante la antesis, incrementándose con el desarrollo de frutos. Benítez-Pardo et al. (2003) señalan que hojas ubicadas en la parte alta del árbol alcanzan concentraciones más altas de $\mathrm{Ca}$, seguido por hojas del estrato medio, mientras que hojas ubicadas en ramas bajas la concentración es mucho menor, de igual manera reportan que hojas más expuestas al sol (lado sur) presentan valores más altos de $\mathrm{Mg}$.

La mayor concentración de $\mathrm{N}$ en 'TA' previo a floración respecto de 'A' puede relacionarse con un efecto de alternancia, Peralta et al. (2014) reportan que en 2010, 'TA' fue el único que mantuvo frutos en el árbol hasta cosecha en todas las unidades experimentales, por tanto, para el 2011 el número de panículas por árbol fue menor que 'A', lo que significa menos puntos de demanda; situación similar para la mayor concentración de $\mathrm{Ca}$ y $\mathrm{Mg}$ en 'A', que exhibió menor rendimiento de frutos; y también puede aplicar para la menor concentración de $\mathrm{Zn}, \mathrm{Fe}, \mathrm{Mn}$ y $\mathrm{Cu}$ en 'TA', que en este caso se atribuye a la mayor demanda del cultivar, ya que en 2010 y 2011 mostró los mayores rendimientos de frutos Peralta et al. (2014).

El decremento de N, en 'TA' previo a cosecha de frutos, corrobora estudios realizados por Medeiros et al. (2005) y Costa et al. (2011) pues apreciaron diferencias en la concentración de $\mathrm{N}$ foliar en cada etapa fenológica del árbol, mayor concentración en la etapa vegetativa y floración, misma que disminuye durante la fructificación y maduración de frutos, por tanto conjeturan que este nutriente se mueve, primeramente a flores y posteriormente a frutos.

Diferencias en las concentraciones de $\mathrm{N}$ y K entre los cultivares podrían estar relacionadas con la capacidad de extracción nutrimental de cada cultivar, Fallas et al. (2010) señalan que Tommy Atkins puede extraer 1065,3 y 1541, 3 mg.kg ${ }^{-1}$ de fruta fresca de $\mathrm{N}$ y $\mathrm{K}$, mientras que Keith extrae 1061,2 y 1587,1 mg.kg ${ }^{-1}$ de fruta fresca de N y K; también Mellado-Vázquez et al. (2012) apreciaron que ‘Tommy Atkins' extrae menor K que el cv. Kent. 
Lo anterior también podría explicar el hecho de que, a pesar de que 'A' y 'MC2' fueron estadísticamente iguales en rendimiento de frutos (Peralta et al., 2014), existieron diferencias entre ellos en las concentraciones de Fe en las dos fechas de muestreo, al respecto, Fallas et al. (2010) encontraron que la concentración de Fe en 'TA' es $241 \%$ mayor que la del cultivar Kent y Mellado-Vázquez et al. (2012) reportan que el cv. Haden puede remover $49 \%$ más Mn que Tommy Atkins.

El Ca y K mostraron comportamientos que difieren con otros estudios, Benitez-Pardo et al. (2003) en el cv. Kent, Medeiros et al. (2005) en Tommy Atkins y Castro-López et al. (2012), en los cultivares Ataulfo, Kent y Tommy Atkins, observaron que desde el inicio de floración hasta la finalización del desarrollo de frutos el $\mathrm{K}$ disminuye y el Ca se incrementa en las hojas, Guzmán et al. (1998) reportan que durante dos años consecutivos realizaron estudios en el cv. Manila en la misma área geográfica donde se desarrolló la presente investigación, en el primer año los valores Ca se incrementaron de floración a fructificación, mientras que en el siguiente año los valores se mantuvieron sin mucha variación e incluso llegaron a disminuir; en el presente estudio el $\mathrm{K}$ se incrementó principalmente en 'TA' y 'A' y la concentración de Ca se mantuvo sin mucha variación.

\section{Fuentes de fertilización}

Respecto de las fuentes de fertilización, el N solo mostró diferencias previo a cosecha, donde lombricompost y bocashi a $10 \mathrm{t} \mathrm{ha}^{-1}$ fueron superiores a lombricompost $5 \mathrm{tha}^{-1}$. En cuanto a $\mathrm{K}$, previo a floración, lombricompost y gallinaza a $10 \mathrm{t} \mathrm{ha}^{-1}$, junto la dosis $230-0-300 \mathrm{~g}$ de NP-K árbol $^{-1}$ exhibieron menores concentraciones que lombricompost a $5 \mathrm{t} \mathrm{ha}^{-1}$. Respecto de $\mathrm{Ca}$, no se apreciaron diferencias entre árboles fertilizados y no fertilizados. En tanto que las concentraciones de $\mathrm{Mg}$ únicamente mostraron diferencias estadísticas antes de la cosecha, donde la dosis 230-0-0 g de $\mathrm{N}-\mathrm{P}-\mathrm{K}$ árbol ${ }^{-1}$ mostró menor concentración que los árboles no fertilizados (Tabla 4). Los resultados obtenidos se asemejan en parte con lo reportado

Tabla 4. Concentración de macronutrientes ( $\mathrm{g} 100 \mathrm{~g}^{-1} \mathrm{~ms}$ ) en hojas de mango, manejados con diferentes fuentes de fertilización, en Veracruz, México, correspondiente al ciclo de producción 2011.

\begin{tabular}{lcccccccc}
\hline \multirow{2}{*}{ Fuente de fertilización } & \multicolumn{4}{c}{ Previo a floración } & \multicolumn{5}{c}{ Previo a cosecha } \\
\cline { 2 - 8 } & $\mathrm{N}$ & $\mathrm{K}$ & $\mathrm{Ca}$ & $\mathrm{Mg}$ & $\mathrm{N}$ & $\mathrm{K}$ & $\mathrm{Ca}$ & $\mathrm{Mg}$ \\
\hline Lombricompost $10 \mathrm{t} \mathrm{ha}^{-1}$ & $1,22 \mathrm{a}$ & $0,94 \mathrm{~b}$ & $0,65 \mathrm{a}$ & $0,09 \mathrm{a}$ & $1,38 \mathrm{a}$ & $1,99 \mathrm{a}$ & $0,59 \mathrm{a}$ & $0,09 \mathrm{ab}$ \\
Bokashi 10 t ha & $1,28 \mathrm{a}$ & $1,16 \mathrm{ab}$ & $0,64 \mathrm{a}$ & $0,09 \mathrm{a}$ & $1,38 \mathrm{a}$ & $1,64 \mathrm{a}$ & $0,72 \mathrm{a}$ & $0,10 \mathrm{ab}$ \\
Gallinaza 10 t ha & $1,39 \mathrm{a}$ & $0,87 \mathrm{~b}$ & $0,60 \mathrm{a}$ & $0,08 \mathrm{a}$ & $1,31 \mathrm{ab}$ & $1,81 \mathrm{a}$ & $0,70 \mathrm{a}$ & $0,08 \mathrm{ab}$ \\
230-0-300 g de N-P-K por árbol & $1,28 \mathrm{a}$ & $0,88 \mathrm{~b}$ & $0,64 \mathrm{a}$ & $0,07 \mathrm{a}$ & $1,30 \mathrm{ab}$ & $1,87 \mathrm{a}$ & $0,60 \mathrm{a}$ & $0,09 \mathrm{ab}$ \\
230-0-0 g de N-P-K por árbol & $1,35 \mathrm{a}$ & $1,03 \mathrm{ab}$ & $0,58 \mathrm{a}$ & $0,08 \mathrm{a}$ & $1,25 \mathrm{ab}$ & $2,02 \mathrm{a}$ & $0,80 \mathrm{a}$ & $0,07 \mathrm{~b}$ \\
Gallinaza 5 t ha $^{-1}$ & $1,30 \mathrm{a}$ & $1,19 \mathrm{ab}$ & $0,66 \mathrm{a}$ & $0,08 \mathrm{a}$ & $1,30 \mathrm{ab}$ & $1,65 \mathrm{a}$ & $0,73 \mathrm{a}$ & $0,08 \mathrm{ab}$ \\
Bokashi 5 t ha $^{-1}$ & $1,30 \mathrm{a}$ & $1,08 \mathrm{ab}$ & $0,65 \mathrm{a}$ & $0,09 \mathrm{a}$ & $1,25 \mathrm{ab}$ & $1,62 \mathrm{a}$ & $0,66 \mathrm{a}$ & $0,10 \mathrm{ab}$ \\
Lombricompost 5 t ha & $1,27 \mathrm{a}$ & $1,45 \mathrm{a}$ & $0,69 \mathrm{a}$ & $0,09 \mathrm{a}$ & $1,23 \mathrm{~b}$ & $2,07 \mathrm{a}$ & $0,72 \mathrm{a}$ & $0,10 \mathrm{ab}$ \\
Testigo & $1,25 \mathrm{a}$ & $1,27 \mathrm{ab}$ & $0,62 \mathrm{a}$ & $0,09 \mathrm{a}$ & $1,25 \mathrm{ab}$ & $1,80 \mathrm{a}$ & $0,68 \mathrm{a}$ & $0,11 \mathrm{a}$ \\
C.V. & 10,8 & 27,5 & 17,1 & 16,9 & 5,9 & 17,8 & 17,7 & 15,1 \\
\hline
\end{tabular}

Columnas con la misma letra son estadísticamente iguales (Tukey $\alpha=0,05$ ).

Tabla 5. Concentración de micronutrientes $\left(\mathrm{mg} \mathrm{kg}^{-1} \mathrm{~ms}\right)$ en hojas de tres cultivares de mango, previo a floración, en Veracruz, México, durante el ciclo de producción 2011.

\begin{tabular}{lcccccccc}
\hline \multirow{2}{*}{ Cultivar } & \multicolumn{4}{c}{ Previo a floración } & \multicolumn{4}{c}{ Previo a cosecha } \\
\cline { 2 - 9 } & $\mathrm{Zn}$ & $\mathrm{Fe}$ & $\mathrm{Mn}$ & $\mathrm{Cu}$ & $\mathrm{Zn}$ & $\mathrm{Fe}$ & $\mathrm{Mn}$ & $\mathrm{Cu}$ \\
\hline Manila Cotaxtla & $29,3 \mathrm{a}$ & $87,3 \mathrm{a}$ & $163,9 \mathrm{a}$ & $19,7 \mathrm{a}$ & $33,5 \mathrm{a}$ & $116,6 \mathrm{a}$ & $71,7 \mathrm{a}$ & $12,5 \mathrm{~b}$ \\
Tommy Atkins & $24,1 \mathrm{~b}$ & $68,6 \mathrm{~b}$ & $88,0 \mathrm{~b}$ & $15,0 \mathrm{~b}$ & $25,3 \mathrm{~b}$ & $63,9 \mathrm{~b}$ & $76,9 \mathrm{a}$ & $13,9 \mathrm{~b}$ \\
Ataulfo & $24,3 \mathrm{~b}$ & $70,0 \mathrm{~b}$ & $109,5 \mathrm{~b}$ & $19,1 \mathrm{a}$ & $26,3 \mathrm{ab}$ & $65,6 \mathrm{~b}$ & $72,3 \mathrm{a}$ & $16,4 \mathrm{a}$ \\
CV & 19,2 & 17,5 & 29,4 & 21,7 & 31,7 & 33,1 & 38,9 & 17,9 \\
\hline
\end{tabular}

Columnas con la misma letra son estadísticamente iguales (Tukey $\alpha=0,05$ ). 
por Silva et al. (2013) que evaluaron en el cultivar Tommy Atkins tres tipos de compostas en dosis de 0,5 y $10 \mathrm{t} \mathrm{ha}^{-1}$, los resultados no mostraron diferencias estadísticas en los contenidos de $\mathrm{N}$, $\mathrm{P}, \mathrm{K}$ y $\mathrm{Ca}$ de las hojas, aunque sí existió en el rendimiento de frutos. Sin embargo, difiere con resultados observados en otros cultivos, Tapia et al. (2014) evaluaron en aguacate Hass la aplicación de fermentos orgánicos, derivados de pescado, composta, microorganismos, lombricompost y lo compararon con una dosis mineral convencional, en los resultados notaron que los fermentos orgánicos mostraron mayor concentración foliar de $\mathrm{N}$ y $\mathrm{K}$ en comparación con la dosis mineral, en cambio en el presente estudio $\mathrm{Ca}$ en ninguno de los muestreos se observó diferencias estadísticas. Los resultados indican que el suelo influyó en el suministro de $\mathrm{N}$, $\mathrm{K}$, Ca y $\mathrm{Mg}$, ya que a pesar de que los fertilizantes orgánicos aportaron fracciones de estos nutrimentos al suelo, los árboles fertilizados con fuente mineral y los no fertilizados suplieron sus necesidades con los disponibles en el suelo, ya que en ningún caso superaron estadísticamente a los no fertilizados, incluso las concentraciones de $\mathrm{Mg}$ en estos fue mayor que una de las fuentes minerales.

Los valores de concentración foliar de $\mathrm{N}$ (1.22-1.38 g $\left.100 \mathrm{~g} \mathrm{~g}^{-1} \mathrm{~ms}\right)$ determinados en el presente estudio, se asemejan a los reportados por Assis et al. (2004), Almeida et al. (2014) y Pinto et al. 2009; supera lo encontrado por Tahir et al. (2003) y es inferior a lo reportado por Benitez-Pardo et al. (2003). En tanto que las concentraciones de K $(0,87$ -

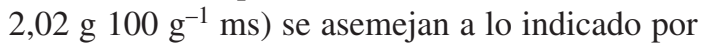
Almeida et al. (2014) y previo a cosecha supera lo reportado por Tahir et al. (2003) y Medeiros et al. (2005). Las concentraciones de $\mathrm{Ca}(0,58-0,73 \mathrm{~g} 100$ $\left.\mathrm{g}^{-1} \mathrm{~ms}\right)$ y $\mathrm{Mg}\left(0,07-0,11 \mathrm{~g} 100 \mathrm{~g}^{-1} \mathrm{~ms}\right)$ son inferiores a lo indicado por Benítez-Pardo et al. (2003), Tahir et al. (2003), Assis et al. (2004), Medeiros et al. (2005), Pinto et al. (2009), Shaaban y Shaaban (2012), Silva et al. (2013) y Almeida et al. (2014). De acuerdo con Quaggio (1996) y Fernandes y Nascimento (2004), las concentraciones de $\mathrm{N}$ son apropiados, $\mathrm{K}$ por arriba del rango óptimo y $\mathrm{Ca}$ y $\mathrm{Mg}$ por debajo del rango óptimo.

En micronutrientes, las concentraciones de $\mathrm{Zn}$ y Fe fueron similares entre árboles fertilizados y no fertilizados en los dos muestreos. Lo que coincide con reportes de Vega y Molina (1999), durante tres años consecutivos fertilizaron árboles de mango del cultivar Tommy Atkins con N (0, 20, 40, 60 y $\left.80 \mathrm{~kg} \mathrm{ha}^{-1}\right)-\mathrm{P}_{2} \mathrm{O}_{5}\left(931,7 \mathrm{~g}\right.$ ábol $\left.^{-1}\right)-\mathrm{K}_{2} \mathrm{O}(714,3 \mathrm{~g}$ árbol $^{-1}$ ), y con Silva et al. (2010), quienes fertilizaron a árboles con diferentes tipos de compostas, en ambos casos se apreció que los valores de $\mathrm{Zn}$ y Fe se comportaron de manera similar con los árboles no fertilizados.

Respecto de Mn, en el primer muestreo las dos dosis minerales 230-0-30 g de N-P-K por árbol fueron iguales a 230-0-0 g de N-P-K por árbol y superior al de los árboles fertilizados de forma orgánica y a los no fertilizados; mientras que, antes de la cosecha lombricompost 10 y 5 tha $^{-1}$ junto con el testigo, fueron inferiores a la dosis 230-0-0 g de N-P-K por árbol y gallinaza $5 \mathrm{t} \mathrm{ha}^{-1}$ (Tabla 6). Los resultados evidencian que los árboles de mango, independientemente de la fuente de nutrición estos extrajeron eficientemente el Mn del suelo, ya que las fuentes minerales a pesar de

Tabla 6. Concentración de micronutrientes $\left(\mathrm{mg} \mathrm{kg}^{-1} \mathrm{~ms}\right)$ en hojas de mango, manejados con diferentes fuentes de fertilización, en Veracruz, México, correspondiente al ciclo de producción 2011.

\begin{tabular}{|c|c|c|c|c|c|c|c|c|}
\hline \multirow{2}{*}{ Cultivar } & \multicolumn{4}{|c|}{ Previo a floración } & \multicolumn{4}{|c|}{ Previo a cosecha } \\
\hline & $\mathrm{Zn}$ & $\mathrm{Fe}$ & $\mathrm{Mn}$ & $\mathrm{Cu}$ & $\mathrm{Zn}$ & $\mathrm{Fe}$ & Mn & $\mathrm{Cu}$ \\
\hline Lombricompost $10 \mathrm{t} \mathrm{ha}^{-1}$ & $26,5 \mathrm{a}$ & $84,1 \mathrm{a}$ & $117,4 \mathrm{~b}$ & $18,4 \mathrm{a}$ & $28,4 \mathrm{a}$ & $65,2 \mathrm{a}$ & $50,2 \mathrm{~b}$ & $12,9 \mathrm{ab}$ \\
\hline Bokashi $10 \mathrm{tha}^{-1}$ & $26,3 \mathrm{a}$ & $81,2 \mathrm{a}$ & $115,5 \mathrm{~b}$ & $18,8 \mathrm{a}$ & $33,0 \mathrm{a}$ & $86,2 \mathrm{a}$ & $69,2 \mathrm{ab}$ & $14,3 \mathrm{ab}$ \\
\hline Gallinaza $10 \mathrm{tha}^{-1}$ & $27,2 \mathrm{a}$ & $68,1 \mathrm{a}$ & $117,5 \mathrm{~b}$ & $18,3 \mathrm{a}$ & $35,8 \mathrm{a}$ & $66,1 \mathrm{a}$ & $70,3 \mathrm{ab}$ & $12,1 \mathrm{ab}$ \\
\hline 230-0-300 g de N-P-K por árbol & $26,6 \mathrm{a}$ & $78,8 \mathrm{a}$ & $190,2 \mathrm{a}$ & $19,2 \mathrm{a}$ & $23,9 \mathrm{a}$ & $65,5 \mathrm{a}$ & $91,3 \mathrm{ab}$ & $11,4 \mathrm{~b}$ \\
\hline 230-0-0 g de N-P-K por árbol & $25,0 \mathrm{a}$ & $77,3 \mathrm{a}$ & $137,8 \mathrm{ab}$ & $18,5 \mathrm{a}$ & $30,9 \mathrm{a}$ & $86,3 \mathrm{a}$ & $110,4 \mathrm{a}$ & $13,9 \mathrm{ab}$ \\
\hline Gallinaza $5 \mathrm{t} \mathrm{ha}^{-1}$ & $29,1 \mathrm{a}$ & $76,2 \mathrm{a}$ & $106,3 \mathrm{~b}$ & $19,7 \mathrm{a}$ & $31,3 \mathrm{a}$ & $102,5 \mathrm{a}$ & $112,1 \mathrm{a}$ & $15,2 \mathrm{ab}$ \\
\hline Bokashi $5 \mathrm{t} \mathrm{ha}^{-1}$ & $25,7 \mathrm{a}$ & $76,2 \mathrm{a}$ & $106,0 \mathrm{~b}$ & $16,8 \mathrm{a}$ & $23,8 \mathrm{a}$ & $79,7 \mathrm{a}$ & $56,4 \mathrm{ab}$ & $15,3 \mathrm{ab}$ \\
\hline Lombricompost $5 \mathrm{t} \mathrm{ha}^{-1}$ & $22,9 \mathrm{a}$ & $70,3 \mathrm{a}$ & $103,5 \mathrm{~b}$ & $17,0 \mathrm{a}$ & $25,4 \mathrm{a}$ & $87,8 \mathrm{a}$ & $52,8 \mathrm{~b}$ & $16,6 \mathrm{a}$ \\
\hline Testigo & $23,8 \mathrm{a}$ & $65,6 \mathrm{a}$ & $89,9 \mathrm{~b}$ & $14,6 \mathrm{a}$ & $22,8 \mathrm{a}$ & $99,0 \mathrm{a}$ & $49,9 \mathrm{~b}$ & $16,6 \mathrm{a}$ \\
\hline C.V. & 19,2 & 17,5 & 29,4 & 21,7 & 31,7 & 33,1 & 38,9 & 17,9 \\
\hline
\end{tabular}

Columnas con la misma letra son estadísticamente iguales (Tukey $\alpha=0,05$ ). 
que no aportaron micronutrimentos, igualaron a las fuentes orgánicas, lo que se atribuye a los adecuados niveles mostrados en la rizosfera de los árboles, similar a lo observado por Vega y Molina (1999), quienes al fertilizar árboles de mango únicamente con fertilizantes minerales apreciaron diferencias en la concentración de Mn, ya que las dosis más altas de nitrógeno indujeron valores que sobrepasaron en un 36\% al testigo. Las concentraciones de Mn a excepción de gallinaza $5 \mathrm{t} \mathrm{ha}^{-1}$, decrecieron en el muestreo realizados previo a cosecha, contrario a lo reportado por Medeiros et al. (2005), Fallas et al. (2010) y Castro-López et al. (2012); lo que puede explicarse por edad de la hoja, ubicación de la hoja muestreada dentro del árbol o presencia de frutos en los brotes muestreados (Chadha et al., 1980; Guzmán et al., 1998; Benitez-Pardo et al., 2003; Tahir et al., 2003; Castro-López et al., 2012).

Las concentraciones de $\mathrm{Cu}$ solo fueron diferentes previo a cosecha, donde la dosis $230-0-300 \mathrm{~g}$ de N-P-K por árbol mostró estadísticamente menores concentraciones que lombricompost $5 \mathrm{t} \mathrm{ha}^{-1}$ y el tratamiento testigo (Tabla 6). Los resultados indican también una eficiente absorción edáfica de $\mathrm{Cu}$, lo que puede estar influenciado directamente por $\mathrm{pH}$ del suelo, ya que al fluctuar entre 6,4 y 6,4 mantuvo a este elemento disponible para las plantas.

De acuerdo con Fernandes y Nascimento (2004) y Singh et al. (2006), las concentraciones de $\mathrm{Zn}$ (22,9-35,8 mg kg-1 ms), Fe (70,3-102,5 mg kg-1 $\mathrm{ms}), \mathrm{Mn}\left(49,9-89,9 \mathrm{mg} \mathrm{kg}^{-1} \mathrm{~ms}\right)$ y $\mathrm{Cu}(11,4-19,7$ $\mathrm{mg} \mathrm{kg}{ }^{-1} \mathrm{~ms}$ ) se encuentran en rangos adecuados.

\section{Conclusión}

La composición nutrimental del follaje presentó variaciones dentro de cada cultivar y entre cultivares. La cantidad de nutrimentos removidos también está influenciado por el cultivar y las condiciones del cultivo. A excepción de $\mathrm{Ca}, \mathrm{Cu}$ y $\mathrm{Mn}$, las fuentes orgánicas y minerales indujeron comportamientos nutrimentales foliares similares.

\section{Literatura Citada}

Almeida, C.X. de; Junior, J.L.P.; Rozane, D.E.; Souza, H.A. de; Hernandes, A.; Natale, W.; Ferraudo, A.S.

2014. Nutrient cycling in mango trees. Semina: Ciências Agrárias, 35: 259-266.

Assis, J.S. de; Silva, D.J.; Moraes, P.L.D. de

2004. Equilíbrio nutricional e distúrbios fisiológicos em manga 'Tommy Atkins'. Revista Brasileira de Fruticultura, 26: 326-329.

Benítez-Pardo, D.; Hernández-Montoya, M.; Osuna-Enciso, T.; Valenzuela-López, M.; Galván-Piña, B.

2003. Muestreo y análisis foliar relacionados con fenología en mango en el sur de Sinaloa, México. Terra Latinoamericana, 21: 273-283.

Castro-López, M.G.; Salazar-García, S.; González-Duran, I.J.L.;

Medina-Torres, R.; González-Valdivia, J.

2012. Evolución nutrimental foliar en tres cultivares de mango en Nayarit, México. Revista Mexicana de Ciencias Agrícolas, 3: 685-700.

Chadha, K.L; Samra, J.S.; Thakur, R.S.

1980. Standardization of leaf-sampling technique for mineral composition of leaves of mango cultivar 'Chausa'. Scientia Horticulturae, 13: 323-329.

Costa, M.E. da; Caldas, A.V.C. e; Oliveira, A. de F.M.; Gurgel, M.T.; Silva, R.M. da

2011. Caracterização nutricional da mangueira "Tommy Atkins" em função da adubação nitrogenada. Agropecuária Científica no Semi-Arido, 7: 16-22.

Guzmán, C.E.; Blanco, S.A.H.; Mosqueda, R.V.; Martínez, A.G. 1998. Variación del contenido foliar de $\mathrm{Ca}, \mathrm{Mg}$ y micronutrimientos en mango cv. Manila. Agronomía Costarricense, 17: 31-37.
Fallas, R.; Bertsch, F.; Miranda, E.; Henríquez, C.

2010. Análisis de crecimiento y absorción de nutrimentos de frutos de mango, cultivares Tommy Atkins y Keith. Agronomía Costarricense, 34: 01-15.

Fernandes, F.M.; Nascimento, V.M. do

2004. Fertilidade do solo e nutrição da mangueira. In: Rozane, D.E.; Darezzo, R.J.; Aguiar, R.L.; Aguilera, G.H.A.; Zambolim, L. Manga: produção integrada, industrialização e comercialização. Viçosa: UFV. 179-198.

Medeiros, A.A. de; Amorim, J.R.A. de; Silva, DJ; Guedes, FX; Guerra, AG; Dantas, JA.

2005. Composição mineral de folhas e exportação de nutrientes pelos frutos de mangueira, em cultivo irrigado, no Rio Grande do Norte. EMPARN. Boletim de Pesquisa e Desenvolvimento, 30. Disponible en: http://ainfo.cnptia.embrapa.br/digital/ bitstream/CPATSA/36387/1/OPB1413.pdf. Consultado: 10/ Abr/2015.

Mellado-Vázquez, A.; Salazar-García, S.; Treviño-de la Fuente, C.A.; González-Durán, I.J.L.; López-Jiménez, A.

2012. Composición y remoción nutrimental de frutos de mango 'Haden' y 'Tommy Atkins' bajo producción forzada. Revista mexicana de ciencias agrícolas, 3: 925-941.

Moyin-Jesu, E.I.; Adeofun, C.O.

2008. Comparative evaluation of different organic fertilizer on the soil fertility, leaf mineral composition, and growth performance of mango seedlings (Magnifera indica L.). Emir. J. Food Agric., 20: 18-30.

Peralta-Antonio, N; Rebolledo-Martínez, A; Becerril-Román,

A.E; Jaén-Contreras, D.; del Angel-Pérez, A.L.

2014. Response to organic fertilization in mango cultivars: Manila, Tommy Atkins and Ataulfo. Journal of soil science and plant nutrition, 14: 688-700. 
Pinto, P.A. da C.; Dias, L.E.; Alvarez, V.V.H.; Choudhury, M.M.; Vieira, G.

2009. Avaliação de estado nutricional da mangueira Tommy Atkins no submédio do vale do rio São Francisco: estabelecimento das normas DRIS. Recursos Rurais, 5: 5-13. Quaggio, J.A.

1996. Adubação e calagem para mangueira e qualidade dos frutos. p. 106-135. In: São A.R. José, I.V.B. Souza, J. Martins Filho, and O.M. Morais (ed.) Manga, tecnologia de produção e mercado. Universidade Estadual do Sudoeste da Bahia, Vitória da Conquista. Pp. 106-135.

Shaaban, S.H.A.; Shaaban, Mahmoud M.

2012. Impact of the nutritional status on yield of nine mango cultivars grown under farm conditions at Giza Governorate, Egypt. Journal of American Science, 8: 304-310.

SAS Institute Inc.

2007.SAS OnlineDoc® 9.2. Cary, NC: SAS Institute Inc.

Silva, A.F.; Mouco, M.A. do C.; Santana, L.M. de; França, C.R.R.S. 2010. Cultivo de mangueira Tommy Atkins com diferentes compostos orgânicos. Embrapa Semiárido. Boletim de Pesquisa e Desenvolvimento, 81. 22 p.
Silva, D.J.; Mouco, M.A. do C.; Gava, C.A.T.; Giongo, V.; Pinto, J.M. 2013. Composto orgânico em mangueiras (Mangifera indica L.) cultivadas no semiárido do nordeste brasileiro. Revista Brasileira de Fruticultura, 35: 875-882.

Singh, V.K.; Bhriguvanshi, S.R.; Reddy, B.M.C.

2006. Nutrition and nutrient management of the mango (Mangifera indica L.) - New thrust for the future perspective. In: Balanced fertilization for sustaining crop productivity, eds. D. K. Bendi, M. S. Brar and S. K. Bansal, Proceedings of the International Symposium held at Punjab. Agricultural University, Luidhiana, India., 333-355.

Tahir, F.M.; Ibrahim, M.; Hamid, K.

2003. Seasonal variation in nutrient concentration of bearing and non-bearing terminals in mango (Mangifera indica $\mathrm{L}$.) Asian Network for Scientific Information, 2: 113-115.

Tapia Vargas, L.M.; Larios Guzmán, A.; Hernández Pérez, A.; Guillén Andrade, $\mathrm{H}$.

2014. Nutrición orgánica del aguacate cv. "Hass" y efecto nutrimental y agronómico. Revista mexicana de ciencias agrícolas, 5: 463-472.

Vega, E.V.; Molina, E.

1999. Fertilización nitrogenada en el cultivo de mango var. Tommy Atkins, en Guanacaste, Costa Rica. Agronomía Costarricense, 23: 37-44. 